\title{
Fast and Accurate Identification of M. tuberculosis Complex Using an Immunochromatographic MPT64 Antigen Detection Test
}

\author{
Anto Jesuraj Uday Kumar ${ }^{1,2 *}$, Hiresave Srinivasa ${ }^{1}$ \\ ${ }^{1}$ Department of Microbiology, St. John's Medical College, Bangalore, India \\ ${ }^{2}$ Infectious Disease Unit, St. John's Research Institute, Bangalore, India \\ Email: *antouday@gmail.com,dr.srinivasa.micro@gmail.com
}

Received 30 September 2015; accepted 4 December 2015; published 7 December 2015

Copyright (C) 2015 by authors and Scientific Research Publishing Inc.

This work is licensed under the Creative Commons Attribution International License (CC BY). http://creativecommons.org/licenses/by/4.0/

(c) (i) Open Access

\begin{abstract}
Background: A new rapid Immunochromatographic test (ICT) kit (MPT64 TB Ag Kit) for detection of MPT64 Antigen in M. tuberculosis (MTB) isolates used for rapid identification of MTB isolates developed by SD (Standard Diagnostics) Bio line, South Korea was evaluated. The ICT is a rapid, reliable and cheaper method that can be used instead of conventional biochemical tests for confirming MTB in culture isolates in resource limited laboratories. The study also evaluated the ability of ICT to detect MPT64-Antigen before the micro MGIT could signal positive. Material/Methods: A total of 450 sputum samples of individual patients were used for the study. 152 isolates of $M y$ cobacteria were recovered from solid and liquid media. These strains were tested for the detection of MPT64-antigen. H37Rv strain was served as the positive reference control and also used for early detection of Antigen experiment. Findings: The development of bands on both test and sample region when H37Rv strain was tested were seen (MPT64 antigen positive). When 138 MTB isolates were tested, it showed a similar banding pattern indicating $100 \%$ sensitivity. MPT64 band formation was not detected in any of the 14 isolates indicating $100 \%$ specificity. Both PPV \& NPV were $100 \%$. All the isolates negative for MPT64 Ag were confirmed as MOTT by conventional biochemical PNBA. The H37Rv strain showed a faint band from the $2^{\text {nd }}$ day onwards from inoculation till $3^{\text {rd }}$ day in the earlier Antigen detection experiment. Conclusion: Rapid identification of MTB culture isolate is a pressing need for diagnosis and proceeding to perform drug susceptibility testing. MPT64 TB Ag detection ICT kit is a rapid, reliable method, good substitute for molecular identification methods, and conventional biochemical test which is time-consuming and technically demanding. The early detection of Antigen can be used as an effective tool in diagnosis.
\end{abstract}

${ }^{*}$ Corresponding author.

How to cite this paper: Kumar, A.J.U. and Srinivasa, H. (2015) Fast and Accurate Identification of M. tuberculosis Complex Using an Immunochromatographic MPT64 Antigen Detection Test. Journal of Tuberculosis Research, 3, 149-156.

http://dx.doi.org/10.4236/itr.2015.34021 


\section{Keywords}

\section{MTB (M. tuberculosis) MOTT (Mycobacteria Other than M. tuberculosis), PNBA (Para Nitro Benzoic Assay), MPT64 Antigen, ICT (Immunochromatography Test), MGIT (Mycobacterium Growth Indicator Tube), SD TB Ag MPT64 Rapid (Standard Diagnostics, Seoul, South Korea)}

\section{Introduction}

Tuberculosis (TB) is an infectious disease caused by the bacillus Mycobacterium tuberculosis (Mtb). It typically affects the lungs (pulmonary TB) but can affect other sites as well (extra pulmonary TB) [1]. The disease is spread in the air when people who are sick with pulmonary TB expel bacteria, by coughing. In general, a relatively small proportion of people infected with Mtb develop TB disease; however, the probability of developing TB disease is higher among people infected with HIV [1]. The 2013 World Health Organization (WHO) estimate showed 9 million (range, 8.6 million - 9.4 million) cases of TB globally. There were 1.5 million TB deaths (little under 1.0 million among HIV-negative people and 0.3 million HIV-associated TB deaths) [2]. In India, about 2.0 - 2.3 million incident cases of TB are reported annually, which account for a fifth of new cases in the world - a greater number than any other country [1]. The situation is further worsened by the increasing number of drug-resistant cases of TB. Thus, there is a need for rapid and correct identification of Mycobacteria and rapid drug sensitivity testing for effective treatment of the disease [3]-[6].

The most common method for diagnosing TB worldwide is sputum smear microscopy (described for more than 100 years) [1]. Culture is the gold standard traditional methods employ solid media, which is less technology-invasive [7]. However, use of solid media takes 4 - 8 weeks to identify growth and a further 4 - 8 weeks if biochemical tests are to be performed. This consequently delays appropriate treatment to patients [8]. Liquid culture methods are widely used by many countries globally, are endorsed by WHO due to its rapid speciation quality and are specific and sensitive as compared to solid culture [7]-[13].

Following recent advances in TB diagnostics, the use of rapid molecular tests for the diagnosis of TB and drug-resistant TB is increasing [1]. Molecular diagnostic technologies are increasingly being used in drug resistance surveys to simplify logistics and reduce laboratory workload. GenoType ${ }^{\circledR}$ MTBDRplus (Hain Lifescience, Germany) and Xpert ${ }^{\circledR}$ MTB/RIF (Cepheid, USA) were used in the national survey in Nigeria completed in 2012. Rifampicin resistance is the most important indicator of MDR-TB, though not a complete surrogate for MDR-TB, particularly in settings where levels of drug resistance are low, with serious clinical implications for affected patients [1].

MPT64, a 24 kDa secretory protein, is one of the major antigens from TB bacteria. Recently, MPT64 has been shown to differentiate the Mycobacterium tuberculosis complex (MTC) from other bacterial species [14]. A mouse monoclonal MPT64 Antibody based kit developed by SD Bio line, South Korea was evaluated for rapid identification of $M$. tuberculosis isolates from other bacterial species (including the BCG strain) and $M y$ cobacterium other than tuberculosis (MOTT) [3]-[6] [15]. The purpose of this study was to evaluate the SD Bio line MPT64 antigen detection test kit for the routine identification of MTB complex against para nitro benzoic acid (PNBA); a biochemical test to differentiate between MTB and MOTT [16]-[19].

In this regard, we evaluated the clinical usefulness, sensitivity, specificity, positive predictive value (PPV), negative predictive value (NPV) \& Kappa of the commercially available Immunochromatographic test kit, SD Tb Ag to MTB complex against para nitro benzoic acid (PNBA); a biochemical test to differentiate between MTB and MOTT.

Moreover, we have also tested the ability of the SD Tb Ag kit to early detect the protein from a liquid medium (MGIT) before the micro MGIT reader could signal the tube positive.

\section{Materials and Methods}

A total of 450 clinically suspected individual TB patient (sputum) samples of 2 - 5 ml volume, were collected from the St. Johns medical college hospital in Karnataka, India.

These samples were subjected to decontamination and homogenization using NaLC-NaOH method for liquid culture and solid culture [20]-[24]. Smears made from concentrated specimens were air dried, heat fixed and the 
smear was covered with carbol fuchsin stain, and Heated until vapour just begins to rise and was allowed to remain on the slide for 5 minutes. After 5 minutes the smear was washed with distilled water, the smear was decolorized using acid alcohol for 2 - 5 minutes or until the smear is sufficiently decolorized, i.e. pale pink. The smear was washed with distilled water and the slide was Covered Methylene blue stain for $1-2$ minute. All the smears were read within 48 hours of preparation using a light microscope (magnification 1000×). They reported the presence or absence of acid-fast bacilli (AFB) using the WHO/IUATLD and RNTCP scale, with a positive result corresponding to $\geq 1$ AFB per 100 high-power fields (HPFs) [21] [25].

Clinical samples after digestion concentration were inoculated in liquid culture (BACTEC MGIT, Beckton Dickinson) and solid culture media (commercial Lowenstein Jenson Medium (LJ)) and were incubated at $37^{\circ} \mathrm{C}$ [21] [23] [24]. MGIT was incubated for six weeks and LJ media up to eight weeks [21] [23] [24]. The culture positive samples were confirmed to be AFB by Ziehl-Neelsen's staining. A total of 152 (Figure 1) culture isolates of Mycobacteria (from liquid and solid culture), using BACTEC MGIT system and Lowenstein Jensen media, were used for the study. ATCC H37Rv strain and Mycobacterium fortuitum were used as the standard positive and negative control respectively.

TB Antigen MPT64 rapid ICT kit, manufactured by Standard Diagnostics, Seoul, South Korea, was used as per the manufacturer's instructions [3]-[6] [15]. Monoclonal mouse antibodies, against MPT64 antigen, are immobilized on a nitrocellulose membrane for confirmation of MTB isolates. The entire test procedure was carried out inside a biosafety class II cabinet and level three biosafety laboratory. $100 \mu \mathrm{l}$ of sample from liquid culture was applied directly without any sample preparation in the sample well. For solid culture isolates 2 - 4 colonies were scraped from the solid medium and suspended in $200 \mu \mathrm{l}$ of extraction buffer provided in the kit. The emulsified solution was applied in the sample well as described above. After 15 minutes of sample application the test was interpreted at room temperature. 5 MGIT were inoculated with reference positive control strain H37Rv ATCC (MTB 27294); $100 \mu l$ media was withdrawn from the tubes next day onwards (after the inoculation), before they were signaled as positive by the fluorescent signals using Micro MGIT and the presence of MPT64 antigen secretion was checked by ICT. The test cassette was compared with positive and negative controls. The development of pink band in control and test region indicated the presence of MPT64 antigen. The positive bands developed within 5 - 10 mins. The sensitivity and specificity of the assay was calculated based on test results from MTBC and MOTT isolates. The absence of band in the control region was considered invalid. All the ICT results were validated by commercially available PNBA (para nitro benzoic assay) test for identification of MTBC or MOTT [16]-[19].

The MPT64 rapid ICT kit and LJ media procured commercially from Standard Diagnostics and Fi-Tech

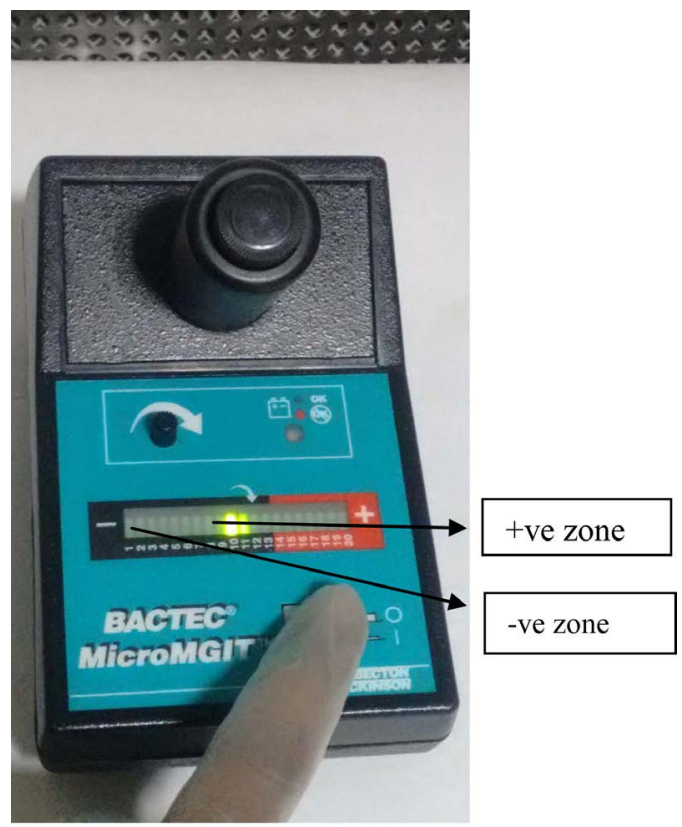

Figure 1. Micro mgit positive and negative zone. 
Chemechtron Pvt. Ltd. Bangalore.

The study was approved by the, St. John's Medical College \& Hospital Institutional Ethical Review Board. All study subjects were enrolled in the study only after they had provided informed written consent.

\section{Statistical Methods Used for Analysis}

\section{Data Were Reported Using Number and Percentages}

The portion of MTB complex was compared between MPT64 Ag and PNBA. Specificity, positive predictive value (PPV), negative predictive value (NPV) \& Kappa of MPT64 was reported. Kappa statistics was used to test the concordance between MPT64 with PNBA. P value less than 5\% was considered stats significant.

\section{Results}

Out of 450 specimens which were cultured 152 (33.7\%) found to be culture positives. These 152 culture positives (liquid culture and solid culture) isolates of Mycobacteria were differentiated using SD MPT64 TB Ag $\mathrm{Kit}^{\circledR}$ assay and PNBA (Table 1). 138 (90\%) of 152 isolates of Mycobacteria were identified as MTBC with Strong positive bands similar to that of H37Rv strain (positive control) regardless of the culture medium (solid or liquid), while no positive signal was observed for any of the 14 MOTT and, indicating a test sensitivity and specificity of $100 \%$ (Table 2) as compared with PNBA. 2 samples that gave faint band on test region were subjected to a repeat test with a longer incubation period of 48 hours as well as by PNBA (para nitro benzoic assay) and found to be MTBC.

There was no significant difference in identification of MTBC isolates between SD MPT64 TB Ag Kit ${ }^{\circledR}$ assay and PNBA. The sensitivity, specificity, positive predictive \& negative predictive values of the SD Ag MPT64 kit was found to be $100 \%$. Kappa statistics value of 1 showed concordance between MPT64 with PNBA (Table 2).

This study also evaluated the ability of SD TB MPT64 Ag Rapid ICT kit to detect antigen before the micro MGIT could signal positive (approximately $10^{5}-10^{6}$ colony forming units (CFU) per ml of medium (Figure 1). The 5 MGIT used for early detection of MPT64 antigen the production of the antigen before the micro MGIT detected positive photograph's (Figure 2). $100 \mu \mathrm{l}$ of the inoculated media withdrawn from the MGIT second day onwards (after the inoculation), was checked for the presence of antigen by means of the band formation in the test region. But the bands were less intense and faint. These samples were re-affirmed after detecting positive to rule out false positivity. The MPT64 antigen detection in the initial stages of all the samples gave faint bands; however, it developed in an intense band from $4^{\text {th }}$ day onwards. The results are shown in table (Table 3 ) (Figure 2) ICT pictorial representation.

Probably modification of the antigen extraction method leading to increased antigen concentration is needed for this rapid test to be used for identification of MTB in clinical samples. The negative test cassettes were ex-

\begin{tabular}{cccc} 
Table 1. Sample distribution/MPT64 results. & & \\
\hline \multirow{2}{*}{ Nature of sample } & \multicolumn{2}{c}{ Numbers tested } & \\
\cline { 2 - 4 } H37Rv & Mpt64 antigen negatives & Mpt64 antigen positive & Total number \\
\hline MTB culture isolates & 0 & 1 & 1 \\
Non tuberculous Mycobacteria isolate & 0 & 138 & 138 \\
Non Mycobacteria isolates & 14 & 0 & 3 \\
Total & 3 & 0 & 14 \\
\hline
\end{tabular}

Table 2. Sensitivity and specificity of SD Bio line TB Ag MPT64 rapid test.

\begin{tabular}{ccccc}
\hline Concordance (kappa) & Sensitivity & Specificity & PPV & NPV \\
\hline $100 \%(1.00)$ & $100 \%$ & $100 \%$ & $100 \%$ & $100 \%$ \\
\hline
\end{tabular}




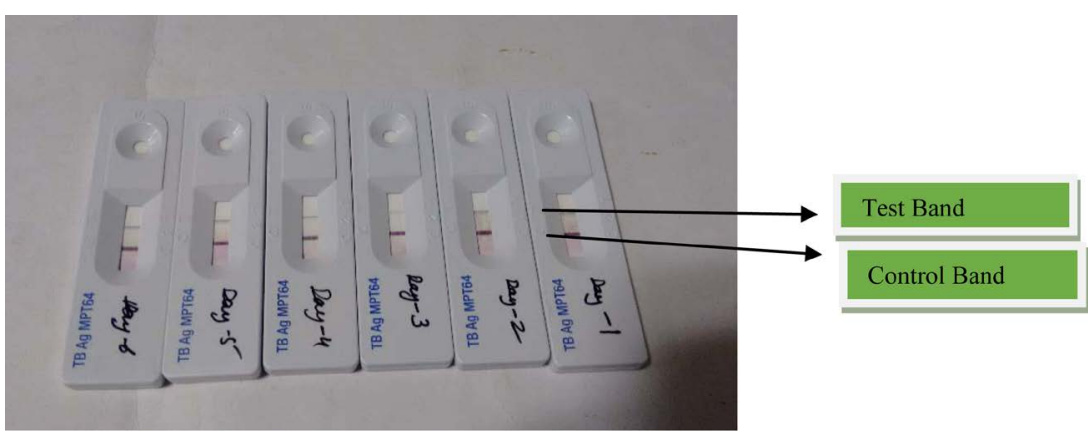

Figure 2. Iidentification of Mycobacterium tuberculosis Complex by MPT64 ICT Kit for early detection experiment.

Table 3. Earlier detection experiment results.

\begin{tabular}{|c|c|c|c|}
\hline \multirow[t]{2}{*}{ Experiment } & \multicolumn{2}{|c|}{$\begin{array}{l}\text { MPT64 antigen } \\
\text { Detection before Micro MGIT instrument signals positive }\end{array}$} & \multirow[t]{2}{*}{$\begin{array}{l}\text { Fluorescent +ve } \\
\text { By Micro MGIT instrument }\end{array}$} \\
\hline & Initial sample & Day of strong band & \\
\hline Sample 1 & Faint band on day 1.2 & $3^{\text {rd }}$ day & $5^{\text {th }}$ day Strong band \\
\hline Sample 2 & Faint band on day 1.2 & $3^{\text {rd }}$ day & $5^{\text {th }}$ day Strong band \\
\hline Sample 3 & Faint band on day 1.2 & $3^{\text {rd }}$ day & $5^{\text {th }}$ day Strong band \\
\hline Sample 4 & Faint band on day 1.2 & $3^{\text {rd }}$ day & $5^{\text {th }}$ day Strong band \\
\hline Sample 5 & Faint band on day 1.2 & $3^{\text {rd }}$ day & $5^{\text {th }}$ day Strong band \\
\hline
\end{tabular}

amined for test band appearance after overnight incubation at room temperature, none of these showed the test band.

\section{Discussion}

A global pandemic disease like Tuberculosis is a major public health problem in a country like India and one of the leading causes of mortality in killing 2 persons every three minutes, nearly 1000 every day [5]. Smear microscopy for AFB is rapid but less sensitivity and cannot discriminate between MTBC and MOTT [1]. Early diagnosis and a well-equipped Mycobacteriology laboratory to perform culture, identification and drug susceptibility testing of Mycobacterium tuberculosis is vital in the management of tuberculosis [1]. The automated liquid systems have improved the recovery of Mycobacterium from smear negative samples [9]-[13], but there is still a need for rapid identification of MTB isolates. Newer rapid identification of the culture isolates and Anti tubercular drug resistant isolates has become a High priority for diagnosis \& patient management [1]. The molecular methods are not cost effective and in a country like India which has a high load of TB disease [1] [5]. A cost effective, rapid \& reliable identification test method will be of enormous help in resource limited settings countries. The Immunochromatographic methods have been found to be ideal diagnostic aid in TB control programme [3]-[6] [15].

The main aim of this study was to evaluate a rapid \& economically feasible test which accurately identifies MTB Complex isolates from positive culture samples. For treatment of Tuberculosis it is necessary to differentiate M. tuberculosis from Mycobacteria other than M. tuberculosis (MOTT). Most of the Mycobacteriology laboratories identify MTB using conventional biochemical tests. These tests are time consuming and laborious. Some useful antigens have been identified by molecular and immunological studies of MTB complex, some of which are very specific to MTB complex. MPT64 also termed as protein Rv1980c is one such antigen [26] [27]. It is a protein secreted by actively growing MTB strains [28]. The MPT64 antigen is absent in BCG strains, $M$. bovis \& M. leprae and in other Mycobacterial species. This has been confirmed by cloning and sequencing of MPT64 gene of H37Rv culture filtrate [29]. The Rapid ICT test can reduce the time of identification drastically compared to conventional biochemical tests [30] [31]. SD MPT64 TB Ag rapid ICT kit was evaluated for Rapid Identification of 152 culture isolates by employing biochemical test PNBA (para nitro benzoic assay) as the ref- 
erence method for MTB identification \& comparative evaluation of the rapid kit. The sensitivity of the rapid test kit was found to be $100 \%$ (138/138). An important observation in this study was no false positive results were detected. Thus the study found the specificity of the rapid ICT test as $100 \%$ \& have concluded that the TB ICT test kit is an useful method for rapid \& routine identification of MTB isolates [30] [31].

The sensitivity, specificity, positive predictive \& negative predictive values of the SD Ag MPT64 kit was found to be $100 \%$. The less technically demanding nature of the ICT coupled with cost effectiveness compared to molecular methods for identification of MTB isolates, makes the rapid ICT method an excellent choice in TB diagnostics [30] [31]. 136 MTB isolates showed similar banding patterns as H37Rv strain. 2 samples which gave a faint band were re-evaluated after further incubation of MGIT. The band formation were sharper and intense and was also confirmed by conventional biochemical test PNBA .None of the MOTT showed band formation for MPT64 antigen, which indicated the specificity of MPT64 antigen to MTB. Previous studies too have shown the absence of this protein in non mycobacterial isolates [29]. The positive \& negative predictive values obtained in the study were excellent with $100 \%$ (Table 3). The PPV of 100\% was observed in a previous study but with a lower NPV (92\%) [15]. The lower NPV was attributed to false negative results. In contrast $100 \%$ NPV (260/260) was detected in one of the study [5]. There are reports of false negativity in ICT methods among few genetically confirmed MTB strains and is attributed to mutation occurring in the specific gene of MTB isolates [30]. There was no difference in the predictive values, sensitivity \& specificity of isolates grown in liquid culture medium and on LJ medium. The intensity of the band was more prominent and sharp with the liquid cultures.

The study also attempted to evaluate the ICT for identification of MPT64 antigen from colonies on LJ media as soon as they were visible and the ability of SD TB MPT64 Ag Rapid ICT kit to detect antigen before the micro MGIT could signal positive. The band appeared in the test region of ICT but was less intense compared to the test done on fully grown colonies. The laboratories from the resource limited areas can speed up the identification process by employing ICT than waiting for full grown colonies which take additional 1 week. The MGIT were manually read using micro MGIT reader from the second day of inoculation and there is a clear demarcation for positive and negative zones (Figure 1). The tubes that were close to positive zone (Figure 1) were vortexed and 100 $\mu$ l of the growth media was checked for MPT64 antigen by ICT and left aside for few minutes. There was band formation on both the test and sample area thought not dark but was clear evidence of antigen presence (Figure 2). From this pilot experiment it appears that MPT64 antigen can be detected from the MGIT just before turning positive or coinciding with flourcent signal using micro MGIT reader. The detection limit was determined to be approximate $105 \mathrm{CFU} / \mathrm{ml}$ by an earlier study [32]. The use of liquid based automated system for the identification of MTB and further drug susceptibility testing is method of choice; however, it is still not widely followed for $\mathrm{Tb}$ diagnosis. Hence in resource limited settings we are endorsing this method and recommend the use of micro MGIT reader to be widely used by Tb laboratories.

Further, we also recommend the use of micro MGIT in clinical microbiology laboratories instead of highly expensive and technically demanding machines like MGIT 320 and 960.

\section{Conclusion}

The SD MPT64 TB Ag Rapid ICT kit is a simple, reliable, rapid and less technology invasive identification kit which can be used as a diagnostic tool in resource limited settings as well as in major diagnostic centres for accurate identification of MTB isolates. ICT contributes in a significant way in Tuberculosis control programmes. The low-cost and rapid PPV \& NPV of the MPT64 antigen detection coupled with high specificity and sensitivity make the ICT a very useful diagnostic tool. India being the high burden Tb country is in need of introducing the ICT and rapid diagnostic methods more than any other country in the world.

\section{Acknowledgements}

This work was supported by St. John's research institute. We thank Dr. George Dsouza, Dr. Dhanasekaran Sivakumaran, Dr. Soham Gupta and Dr. Salesh Chandran for their valuable inputs about the article and the staff of Microbiology Counter, St. John’s Medical College Hospital.

\section{Competing Interests}

The authors declare that they have no competing interests. 


\section{References}

[1] WHO (2014) Global Global Tuberculosis Report 2014.

[2] WHO (2013) HIV-Associated TB Facts 2013.

[3] TB Antigen MPT64. http://www.standardia.com/en/home/product/rapid/infectious-disease/TB_Ag_MPT64.html

[4] Kanade, S., Nataraj, G., Suryawanshi, R. and Mehta, P. (2012) Utility of MPT64 Antigen Detection Assay for Rapid Characterization of Mycobacteria in a Resource Constrained Setting. Indian Journal of Tuberculosis, 59, 92-96.

[5] Kumar, V.G., Urs, T.A. and Ranganath, R.R. (2011) MPT64 Antigen Detection for Rapid Confirmation of M. Tuberculosis Isolates. BMC Research Notes, 4, 79. http://dx.doi.org/10.1186/1756-0500-4-79

[6] Hasegawa, N., Miura, T., Ishii, K., Yamaguchi, K., Lindner, T.H., Merritt, S., Matthews, J.D. and Siddiqi, S.H. (2002) New Simple and Rapid Test for Culture Confirmation of Mycobacterium Tuberculosis Complex: A Multicenter Study. Journal of Clinical Microbiology, 40, 908-912. http://dx.doi.org/10.1128/JCM.40.3.908-912.2002

[7] WHO (2006) International Standards for Tuberculosis Care—Diagnosis, Treatment, Public Health.

[8] World Health Organization (2007) Use of Liquid TB Culture and Drug Susceptibility Testing (DST) in Low and Medium Income Settings. Summary Report of the Expert Group Meeting on the Use of Liquid Culture Media. Geneva.

[9] Chihota, V.N., Grant, A.D., Fielding, K., Ndibongo, B., van Zyl, A., Muirhead, D., et al. (2010) Liquid vs. Solid Culture for Tuberculosis: Performance and Cost in a Resource-Constrained Setting. International Journal of Tuberculosis and Lung Disease, 14, 1024-1031.

[10] Cornfield, D.B., Beavis, K.G., Greene, J.A., Bojak, M. and Bondi, J. (1997) Mycobacterial Growth and Bacterial Contamination in the Mycobacteria Growth Indicator Tube and BACTEC 460 Culture Systems. Journal of Clinical Microbiology, 35, 2068-2071.

[11] Leitritz, L., Schubert, S., Bucherl, B., Masch, A., Heesemann, J. and Roggenkamp, A. (2001) Evaluation of BACTEC MGIT 960 and BACTEC 460TB Systems for Recovery of Mycobacteria from Clinical Specimens of a University Hospital with Low Incidence of Tuberculosis. Journal of Clinical Microbiology, 39, 3764-3767. http://dx.doi.org/10.1128/JCM.39.10.3764-3767.2001

[12] Pfyffer, G.E., Welscher, H.-M., Kissling, P., Cieslak, C., Casal, M.J., Gutierrez, J. and Rusch-Gerdes, S. (1997) Comparison of the Mycobacteria Growth Indicator Tube (MGIT) with Radiometric and Solid Culture for Recovery of Acidfast Bacilli. Journal of Clinical Microbiology, 35, 364-368.

[13] Cruciani, M., Scarparo, C., Malena, M., Bosco, O., Serpelloni, G. and Mengoli, C. (2004) Meta-Analysis of BACTEC MGIT 960 and BACTEC 460 TB, with or without Solid Media, for Detection of Mycobacteria. Journal of Clinical Microbiology, 42, 2321-2325. http://dx.doi.org/10.1128/JCM.42.5.2321-2325.2004

[14] Tomiyama, T., Matsuo, K. and Abe, C. (1997) Rapid Identification of Mycobacterium tuberculosis by an Immunochromatography Using Anti-MPB64 Monoclonal Antibodies. The International Journal of Tuberculosis and Lung Disease: The Official Journal of the International Union against Tuberculosis and Lung Disease, 1, S59.

[15] Ismail, N.A., Baba, K., Pombo, D. and Hoosen, A.A. (2009) Use of an Immunochromatographic Kit for the Rapid Detection of Mycobacterium tuberculosis from Broth Cultures. International Journal of Tuberculosis and Lung Disease, 13, 1045-1047.

[16] Giampaglia, C.M.S., Martins, M.C., Chimara, E., et al. (2007) Differentiation of Mycobacterium tuberculosis from Other Mycobacteria with Rho-Nitrobenzoic Acid Using MGIT960. International Journal of Tuberculosis and Lung Disease, 11, 803-807.

[17] Tsukamura, M. (1981) A Review of the Methods of Identification and Differentiation of Mycobacteria. Clinical Infectious Diseases, 3, 841-851. http://dx.doi.org/10.1093/clinids/3.5.841

[18] Tsukamura, M. and Tsukamura, S. (1964) Differentiation of Mycobacterium tuberculosis and Mycobacterium bovis by P-Nitrobenzoic Acid Susceptibility. Tubercle, 45, 64-65.

[19] Collins, T. and Levett, P.N. (1989) Radiometric Studies on the Use of Selective Inhibitors in the Identification of Mycobacterium spp. Journal of Medical Microbiology, 30, 175-181.

[20] Schaaf HS, Zumla A. (2009) Tuberculosis: A Comprehensive Clinical Reference: Elsevier Health Sciences.

[21] RNTCP (2011) Status TB Report, 2010. New Delhi: Central TB Division. Directorate General of Health Services, Ministry of Health and Family Welfare. www.tbcindia.org

[22] Becton, Dickinson and Company (2004) BACTEC MGIT 960 System User’s Manual. Sparks, MA.

[23] MGIT for BACTECTM (2007) MGIT 960"тм TB System Manual.

[24] Forbes, B.A., Sahm, D.F. and Weissfeld, A.S. (2007) Bailey \& Scott’s Diagnostic Microbiology. 12th Edition, Elsevier, Amsterdam.

[25] World Health Organization (1998) Laboratory Services in Tuberculosis Control. WHO/Tuberculosis/98.258, WHO, 
Geneva.

[26] Wang, Z.H., Potter, B.M., Gray, A.M., Sacksteder, K.A., Geisbrecht, B.V. and Laity, J.H. (2007) The Solution Structure of Antigen MPT64 from Mycobacterium tuberculosis Defines a New Family of Beta-Grasp Proteins. Journal of Molecular Biology, 366, 375-381. http://dx.doi.org/10.1016/j.jmb.2006.11.039

[27] Yamaguchi, R., Matsuo, K., Yamazaki, A., Abe, C., Nagai, S., Terasaka, K. and Yamada, T. (1989) Cloning and Characterization of the Gene for Immunogenic Protein MPB64 of Mycobacterium bovis BCG. Infection and Immunity, 57, 283-288.

[28] Oettinger, T. and Andersen, A.B. (1994) Cloning and B-Cell-Epitope Mapping of MPT64 from Mycobacterium tuberculosis H37Rv. Infection and Immunity, 62, 2058-2064.

[29] Gennaro, M.L. (2000) Immunologic Diagnosis of Tuberculosis. Clinical Infectious Diseases, 30, $243-246$.

[30] Hillemann, D., Rusch-Gerdes, S. and Richter, E. (2005) Application of the Capilia TB Assay for Culture Confirmation of Mycobacterium tuberculosis Complex Isolates. International Journal of Tuberculosis and Lung Disease, 9, 14091411.

[31] Ngamlert, K., Sinthuwattanawibool, C., McCarthy, K.D., Sohn, H., Starks, A., Kanjanamongkolsiri, P., et al. (2009) Diagnostic Performance and Costs of Capilia TB for Mycobacterium tuberculosis Complex Identification from BrothBased Culture in Bangkok, Thailand. Tropical Medicine and International Health, 14, 748-753. http://dx.doi.org/10.1111/j.1365-3156.2009.02284.X

[32] Park, M.Y., Kim, Y.J., Hwang, S.H., Kim, H.H., Lee, E.Y., Jeong, S.H., et al. (2009) Evaluation of an Immunochromatographic Assay Kit for Rapid Identification of Mycobacterium tuberculosis Complex in Clinical Isolates. Journal of Clinical Microbiology, 47, 481-484. http://dx.doi.org/10.1128/JCM.01253-08

\section{Abbreviation}

Tuberculosis (TB), Mycobacterium tuberculosis (Mtb), World Health Organization (WHO), human immunodeficiency virus (HIV), Multi-drug-resistant tuberculosis (MDR-TB), MTB (M. tuberculosis), MTC ((Mycobacterium tuberculosis complex), MOTT (mycobacteria other than $M$. tuberculosis), PNBA (para nitro benzoic assay), MPT64 antigen, ICT (Immunochromatography test), MGIT (mycobacterium growth indicator tube), positive predictive value (PPV), negative predictive value (NPV). Standard Diagnostics (SD Ag MPT64 kit), GenoType ${ }^{\circledR}$ MTBDRplus (Hain Lifescience, Germany), multidrug-resistant tuberculosis (MDR-TB). GeneXpert or Xpert TB Test. TB Diagnosis and Resistance Testing. 